\title{
Probing the muon $g-2$ anomaly with the Higgs boson at a muon collider
}

\author{
Dario Buttazzo ${ }^{1}$ and Paride Paradisi $\odot^{2,3}$ \\ ${ }^{1}$ Istituto Nazionale di Fisica Nucleare, Sezione di Pisa, I-56127 Pisa, Italy \\ ${ }^{2}$ Dipartimento di Fisica e Astronomia "G. Galilei," Università di Padova, 35131 Padova, Italy \\ ${ }^{3}$ Istituto Nazionale Fisica Nucleare, Sezione di Padova, I-35131 Padova, Italy
}

(Received 10 December 2020; revised 5 May 2021; accepted 13 September 2021; published 14 October 2021)

\begin{abstract}
We point out that heavy new physics contributions in leptonic dipole moments and high-energy cross sections of lepton pairs into Higgs bosons and photons are connected model-independently. In particular, we demonstrate that a muon collider, running at center-of-mass energies of several $\mathrm{TeV}$, can provide a unique test of new physics in the muon $g-2$ through the study of high-energy processes such as $\mu^{+} \mu^{-} \rightarrow h \gamma$. This high-energy test would be of the utmost importance to shed light on the longstanding muon $g-2$ anomaly as it is not affected by the hadronic and experimental uncertainties entering the current low-energy determination of the muon $g-2$. Furthermore, we show that the current bound on the muon electric dipole moment can be improved by three orders of magnitude, down to few $\times 10^{-22} e \mathrm{~cm}$.
\end{abstract}

DOI: $10.1103 /$ PhysRevD.104.075021

\section{INTRODUCTION}

The anomalous magnetic moment of the muon has provided, over the last ten years, an enduring hint for new physics (NP). The experimental value of $a_{\mu}=\left(g_{\mu}-2\right) / 2$ from the E821 experiment at BNL [1] was recently confirmed by the E989 experiment at Fermilab [2], yielding the experimental average $a_{\mu}^{\mathrm{EXP}}=116592061(41) \times 10^{-11}$. The comparison of this value with the Standard Model (SM) prediction $a_{\mu}^{\mathrm{SM}}=116591810(43) \times 10^{-11}$ [3] shows an interesting $4.2 \sigma$ discrepancy [2]

$$
\Delta a_{\mu}=a_{\mu}^{\mathrm{EXP}}-a_{\mu}^{\mathrm{SM}}=251(59) \times 10^{-11} .
$$

The forthcoming runs of the E989 experiment plan to reduce the experimental uncertainty by a factor of four. Moreover, a completely new low-energy approach to measuring the muon $g-2$ is being developed by the E34 collaboration at J-PARC [4]. On the theory side, there is also an ongoing effort to reduce the leading SM uncertainty stemming from hadronic corrections [5].

Given the difficulty of controlling all these effects at the required level of precision, we think it is crucial to have an independent test of NP in the muon $g-2$, not affected by the hadronic and experimental uncertainties entering the current low-energy determination of the muon $g-2$.

Published by the American Physical Society under the terms of the Creative Commons Attribution 4.0 International license. Further distribution of this work must maintain attribution to the author(s) and the published article's title, journal citation, and DOI. Funded by SCOAP ${ }^{3}$.
Incidentally, the observed muon $g-2$ discrepancy can be accommodated by a NP effect of the same size as the $\mathrm{SM}$ weak contribution $\sim 5 G_{\mathrm{F}} m_{\mu}^{2} / 24 \sqrt{2} \pi^{2} \approx 2 \times 10^{-9}$ [3]. Therefore, a very natural explanation of Eq. (1) could be achieved within weakly interacting NP scenarios emerging at a scale $\Lambda$ close to the electroweak scale. Remarkably, this possibility could be connected with the solution of the hierarchy problem and could provide, at the same time, a WIMP dark matter candidate. Unfortunately, the lack for new particles at LEP and LHC strongly disfavors this interpretation. As a result, two possibilities seem to emerge to solve the muon $g-2$ anomaly while avoiding the stringent LEP and LHC bounds. Either NP is very light $(\Lambda \lesssim 1 \mathrm{GeV})$ and feebly coupled to SM particles, see e.g., [9], or NP is very heavy $(\Lambda \gg 1 \mathrm{TeV})$ and strongly coupled. Here, we take the second direction.

Heavy NP contributions to the muon $g-2$ arise from the dimension-6 dipole operator $\left(\bar{\mu}_{L} \sigma_{\mu \nu} \mu_{R}\right) H F^{\mu \nu}$ [10] where $H=v+h / \sqrt{2}$ contains both the Higgs boson field $h$ and its vacuum expectation value $v=174 \mathrm{GeV}$ and $F^{\mu \nu}$ is the electromagnetic field strenght tensor. After electroweak symmetry breaking $H \rightarrow v$ and we obtain the prediction $\Delta a_{\mu}^{\mathrm{NP}} \sim\left(g_{\mathrm{NP}}^{2} / 16 \pi^{2}\right) \times\left(m_{\mu} v / \Lambda^{2}\right)$, where $g_{\mathrm{NP}}$ is the typical coupling of the NP sector. Therefore, the NP chiral enhancement $v / m_{\mu} \sim 10^{3}$ with respect to the SM weak contribution, together with the assumption of a new strong dynamics with $g_{\mathrm{NP}} \sim 4 \pi$, bring the sensitivity of the muon $g-2$ to NP scales of order $\Lambda \sim 100 \mathrm{TeV}$ [11].

Directly detecting new particles at such high scales is far beyond the capabilities of any foreseen collider. Moreover, even assuming the discovery of new particles by their direct production [12], it would be very hard, if not impossible, to 
unambiguously associate them to $\Delta a_{\mu}$. In other words, it would be desirable to test the muon $g-2$ anomaly modelindependently.

In this work, we argue that a muon collider (MC) running at energies $E$ of several $\mathrm{TeV}$ would represent the only machine enabling to probe NP in the muon $g-2$ in a completely model-independent way. Indeed, the very same dipole operator that generates $\Delta a_{\mu}$ unavoidably induces also a NP contribution to the scattering process $\mu^{+} \mu^{-} \rightarrow h \gamma$. Measuring the cross section for this process would thus be equivalent to measuring $\Delta a_{\mu}$. This would however be a direct determination of the NP contribution, not hampered by the hadronic uncertainties that affect the SM prediction of $a_{\mu}$.

At first sight, it could seem impossible to be sensitive to such a tiny value of $\Delta a_{\mu} \sim 10^{-9}$ at a collider experiment. However, analogously to the case of weak interaction cross sections in the effective Fermi theory, the cross section for $\mu^{+} \mu^{-} \rightarrow h \gamma$ as induced by the effective dipole operator grows with the square of the collider energy. As a result, a high-energy measurement with $\mathcal{O}(1)$ precision will be sufficient to disentangle NP effects from the SM background. This is the first example in high-energy particle physics of a sensitivity to a magnetic moment at this level, several orders of magnitude below all the other current and projected collider constraints. In order to reach such tiny values of $\Delta a_{\mu}$ it is however crucial to accelerate the muon pairs to the highest possible multi-TeV energies.

We stress that our results are valid for $E \ll \Lambda$ where the effective field theory (EFT) description is justified.

A high-energy MC with the luminosity needed for particle physics experiments [13] is currently not feasible. Nevertheless, several efforts to overcome the technological challenges are ongoing [14], and it is crucial to explore the broad physics potential of such a machine in order to pave the road for the forthcoming accelerator and detector studies. A MC is the ideal machine to search for NP at the highest possible energies, both directly and indirectly. Indeed, muons could in principle be accelerated to multi$\mathrm{TeV}$ energies, as their larger mass greatly suppresses synchrotron radiation compared to the electron-positron case. Furthermore, the physics reach of the MC overtakes that of a proton-proton collider of the same energy since all of the beam energy is available for the hard collision, compared to the fraction of the proton energy carried by the partons: a $\mathrm{MC}$ in the $10 \mathrm{TeV}$ range has roughly the same energy available for hard scatterings as a $100 \mathrm{TeV}$ hadron collider [13].

The physics case of a high-energy determination of $\Delta a_{\mu}$, which is unique of a MC, represents a striking example of the complementarity and interplay of the high-energy and high-intensity frontiers of particle physics. At the same time, it highlights the far reaching potential of a MC, that offers a new powerful way to probe NP which is complementary both to direct searches for new particles, and to the indirect tests conducted at low energy through highprecision experiments.

The paper is organized as follows. In Sec. II, we introduce the SM effective field theory (SMEFT), containing operators up to dimension-6, contributing to $a_{\ell}$. After performing a one-loop calculation of $a_{\ell}$ in such EFT, in Sec. III, we study the high-energy processes at a MC which are sensitive to the same NP effects entering $a_{\ell}$. In Sec. IV, we comment on the possibility of measuring the rare Higgs decays $h \rightarrow \ell^{+} \ell^{-} \gamma$ (with $\ell=\mu, \tau$ ) that are induced by the same dipole operator generating $a_{\ell}$. The huge number of Higgs bosons that could be produced at a MC [15] could in principle allow the measurement of these rare processes, and thus the extraction of $a_{\ell}$.

\section{THE MUON $g-2$ IN THE SMEFT}

New interactions emerging at a scale $\Lambda$ larger than the electroweak scale can be described at energies $E \ll \Lambda$ by an effective Lagrangian containing nonrenormalizable $S U(3)_{c} \otimes S U(2)_{L} \otimes U(1)_{Y}$ invariant operators. Focusing on the leptonic $g-2$, the relevant effective Lagrangian contributing to them, up to one-loop order, reads [10]

$$
\begin{aligned}
\mathcal{L}= & \frac{C_{e B}^{\ell}}{\Lambda^{2}}\left(\bar{\ell}_{L} \sigma^{\mu \nu} e_{R}\right) H B_{\mu \nu}+\frac{C_{e W}^{\ell}}{\Lambda^{2}}\left(\bar{\ell}_{L} \sigma^{\mu \nu} e_{R}\right) \tau^{I} H W_{\mu \nu}^{I} \\
& +\frac{C_{T}^{\ell}}{\Lambda^{2}}\left(\bar{\ell}_{L}^{a} \sigma_{\mu \nu} e_{R}\right) \varepsilon_{a b}\left(\bar{Q}_{L}^{b} \sigma^{\mu \nu} u_{R}\right)+\text { H.c. }
\end{aligned}
$$

where it is assumed that the NP scale $\Lambda \gtrsim 1 \mathrm{TeV}$. The Feynman diagrams relevant for the leptonic $g-2$ are displayed in Fig. 1. They lead to the following result

$$
\begin{aligned}
\Delta a_{\ell} \simeq & \frac{4 m_{\ell} v}{e \Lambda^{2}}\left(C_{e \gamma}^{\ell}-\frac{3 \alpha}{2 \pi} \frac{c_{W}^{2}-s_{W}^{2}}{s_{W} c_{W}} C_{e Z}^{\ell} \log \frac{\Lambda}{m_{Z}}\right) \\
& -\sum_{q=c, t} \frac{4 m_{\ell} m_{q}}{\pi^{2}} \frac{C_{T}^{\ell q}}{\Lambda^{2}} \log \frac{\Lambda}{m_{q}}
\end{aligned}
$$

where $s_{W}, c_{W}$ are the sine and cosine of the weak mixing angle, $\quad C_{e \gamma}=c_{W} C_{e B}-s_{W} C_{e W}$ and $C_{e Z}=-s_{W} C_{e B}-$ $c_{W} C_{e W}$. Additional loop contributions from the operators $H^{\dagger} H W_{\mu \nu}^{I} W^{I \mu \nu}, H^{\dagger} H B_{\mu \nu} B^{\mu \nu}$, and $H^{\dagger} \tau^{I} H W_{\mu \nu}^{I} B^{\mu \nu}$ are suppressed by the lepton Yukawa couplings and can be neglected. Moreover, in Eq. (3), we assumed for simplicity that $C_{e B}, C_{e W}$ and $C_{T}$ are real. Since only the first two operators of Eq. (2) generate electromagnetic dipoles at tree-level, we include their one-loop renormalization effects to $C_{e \gamma}^{\ell}$

$C_{e \gamma}^{\ell}\left(m_{\ell}\right) \simeq C_{e \gamma}^{\ell}(\Lambda)\left(1-\frac{3 y_{t}^{2}}{16 \pi^{2}} \log \frac{\Lambda}{m_{t}}-\frac{4 \alpha}{\pi} \log \frac{m_{t}}{m_{\ell}}\right)$.

In order to see where we stand, let us determine the NP scale probed by $\Delta a_{\ell}$. From Eq. (3) we find that 


$$
\begin{aligned}
\frac{\Delta a_{\mu}}{3 \times 10^{-9}} \approx & \left(\frac{250 \mathrm{TeV}}{\Lambda}\right)^{2} \\
& \times\left(C_{e \gamma}^{\mu}-0.2 C_{T}^{\mu t}-0.001 C_{T}^{\mu c}-0.05 C_{e Z}^{\mu}\right) .
\end{aligned}
$$

A few comments are in order:

(i) The $\Delta a_{\mu}$ discrepancy can be solved for a NP scale up to $\Lambda \approx 250 \mathrm{TeV}$. This requires a strongly coupled NP sector where $C_{e \gamma}^{\mu}$ and/or $C_{T}^{\mu t} \sim g_{\mathrm{NP}}^{2} / 16 \pi^{2} \sim 1$ and a chiral enhancement $v / m_{\mu}$ compared with the weak SM contribution [16]. For such large values of $\Lambda$ direct NP particle production is beyond the reach of any foreseen collider. However, as we shall see, the physics responsible for $\Delta a_{\mu}$ can still be tested through high-energy processes such as $\mu^{+} \mu^{-} \rightarrow h \gamma$ or $\mu^{+} \mu^{-} \rightarrow q \bar{q}$ (with $q=c, t$ ).

(ii) If the underlying NP sector is weakly coupled, $g_{\mathrm{NP}} \lesssim 1$, then $C_{e \gamma}^{\mu}$ and $C_{T}^{\mu t} \lesssim 1 / 16 \pi^{2}$, implying $\Lambda \lesssim$ $20 \mathrm{TeV}$ to solve the $\Delta a_{\mu}$ anomaly. In this case, a MC could still be able to directly produce NP particles [12]. Yet, the study of the processes $\mu^{+} \mu^{-} \rightarrow h \gamma$ and $\mu^{+} \mu^{-} \rightarrow q \bar{q}$ could be crucial to reconstruct the effective dipole vertex $\mu^{+} \mu^{-} \gamma$.

(iii) If the NP sector is weakly coupled, and further $\Delta a_{\mu}$ scales with lepton masses as the SM weak contribution, then $\Delta a_{\mu} \sim m_{\mu}^{2} / 16 \pi^{2} \Lambda^{2}$. Here, the experimental value of $\Delta a_{\mu}$ can be accommodated only provided that $\Lambda \lesssim 1 \mathrm{TeV}$. For such a low NP scale the EFT description breaks down at the typical multi-TeV MC energies, and new resonances cannot escape from direct production.

\section{HIGH-ENERGY PROBES OF THE MUON $\boldsymbol{g}-\mathbf{2}$}

The main contribution to $\Delta a_{\mu}$ comes from the dipole operator $O_{e \gamma}=\left(\bar{\ell}_{L} \sigma_{\mu \nu} e_{R}\right) H F^{\mu \nu}$ when after electroweak symmetry breaking $H \rightarrow v$. The same operator also induces a contribution to the process $\mu^{+} \mu^{-} \rightarrow h \gamma$ that grows with energy (see Fig. 1), and thus can become dominant over the SM cross section at a very high-energy collider. Assuming that $m_{h} \ll \sqrt{s}$, which is an excellent approximation at a $\mathrm{MC}$, we find the following differential cross section

$$
\frac{d \sigma_{h \gamma}}{d \cos \theta}=\frac{\left|C_{e \gamma}^{\mu}\right|^{2}}{\Lambda^{4}} \frac{s}{64 \pi}\left(1-\cos ^{2} \theta\right)
$$

where $\cos \theta$ is the photon scattering angle. Notice that there is an identical contribution also to the process $\mu^{+} \mu^{-} \rightarrow Z \gamma$ since $H$ contains the longitudinal polarizations of the $Z$. The total $\mu^{+} \mu^{-} \rightarrow h \gamma$ cross section is

$$
\sigma_{h \gamma}=\frac{s}{48 \pi} \frac{\left|C_{e \gamma}^{\mu}\right|^{2}}{\Lambda^{4}} \approx 0.7 \mathrm{ab}\left(\frac{\sqrt{s}}{30 \mathrm{TeV}}\right)^{2}\left(\frac{\Delta a_{\mu}}{3 \times 10^{-9}}\right)^{2}
$$
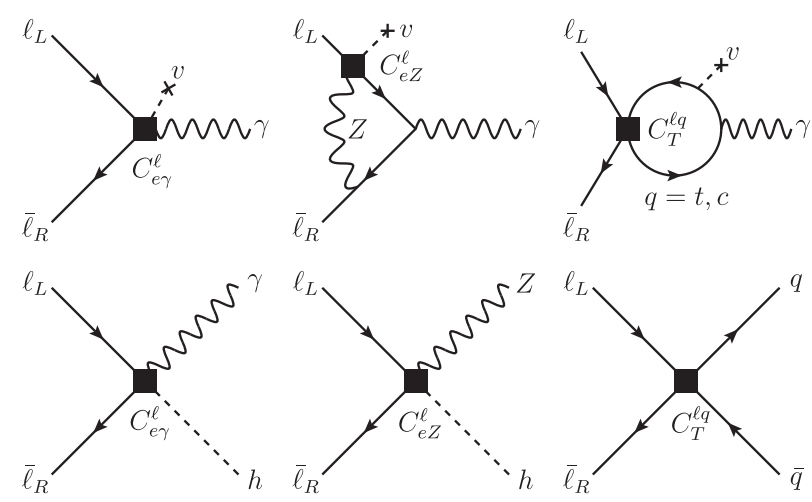

FIG. 1. Upper row: Feynman diagrams contributing to the leptonic $g-2$ up to one-loop order in the Standard Model EFT. Lower row: Feynman diagrams of the corresponding high-energy scattering processes. Dimension-6 effective interaction vertices are denoted by a square.

where in the last equation we assumed no contribution to $\Delta a_{\mu}$ other than the one from $C_{e \gamma}^{\mu}$. Moreover, we included running effects for $C_{e \gamma}^{\mu}$, see Eq. (4), from a scale $\Lambda \approx 100 \mathrm{TeV}$. Given the scaling with energy of the reference integrated luminosity [13]

$$
\mathcal{L}=\left(\frac{\sqrt{s}}{10 \mathrm{TeV}}\right)^{2} \times 10 \mathrm{ab}^{-1}
$$

one gets about 60 total $h \gamma$ events at $\sqrt{s}=30 \mathrm{TeV}$.

The SM irreducible $\mu^{+} \mu^{-} \rightarrow h \gamma$ background is small. The dominant contribution arises at one-loop [18] due to the muon Yukawa coupling suppression of the tree-level part, $\sigma_{h \gamma}^{\mathrm{SM}} \approx 2 \times 10^{-2} \mathrm{ab}\left(\frac{30 \mathrm{TeV}}{\sqrt{s}}\right)^{2}$, and can be neglected for $\sqrt{s} \gg \mathrm{TeV}$. The main source of background comes from $Z \gamma$ events, where the $Z$ boson is incorrectly reconstructed as a Higgs. This cross section is large, due to the contribution from transverse polarizations,

$$
\frac{d \sigma_{Z \gamma}}{d \cos \theta}=\frac{\pi \alpha^{2}}{4 s} \frac{1+\cos ^{2} \theta}{\sin ^{2} \theta} \frac{1-4 s_{W}^{2}+8 s_{W}^{4}}{s_{W}^{2} c_{W}^{2}} .
$$

There are two ways to isolate the $h \gamma$ signal from the background: by means of the different angular distributions of the two processes - the SM $Z_{\gamma}$ peaks in the forward region, while the signal is central-and by accurately distinguishing $h$ and $Z$ bosons from their decay products, e.g., by precisely reconstructing their invariant mass.

To estimate the reach on $\Delta a_{\mu}$ we consider a cut-andcount experiment in the $b \bar{b}$ final state, which has the highest signal yield (with branching ratios $\mathcal{B}(h \rightarrow b \bar{b})=0.58$, $\mathcal{B}(Z \rightarrow b \bar{b})=0.15)$. The significance of the signaldefined as $N_{S} / \sqrt{N_{B}+N_{S}}$, with $N_{S, B}$ the number of signal and background events-is maximized in the central region $|\cos \theta| \lesssim 0.6$. At $30 \mathrm{TeV}$ one gets 


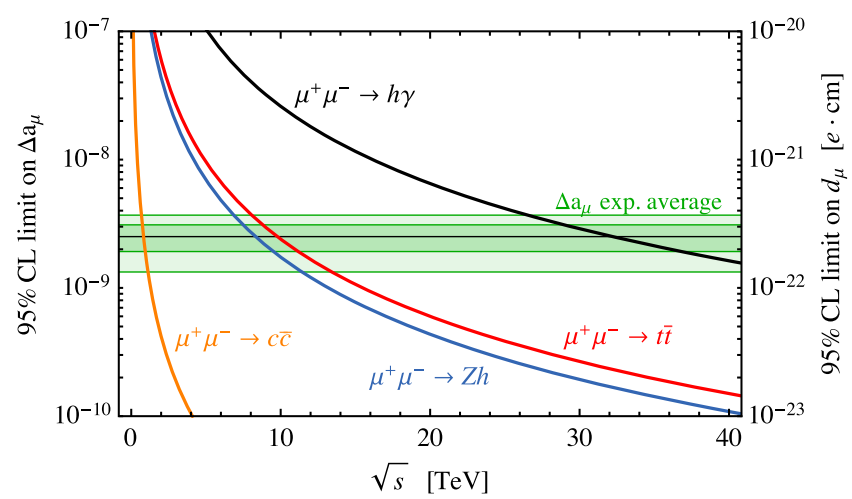

FIG. 2. $95 \%$ C.L. reach on the muon anomalous magnetic moment $\Delta a_{\mu}$, as well as on the muon $\operatorname{EDM} d_{\mu}$, as a function of the collider center-of-mass energy $\sqrt{s}$, from the processes $\mu^{+} \mu^{-} \rightarrow h \gamma$ (black), $\mu^{+} \mu^{-} \rightarrow h Z$ (blue), $\mu^{+} \mu^{-} \rightarrow t \bar{t}$ (red), and $\mu^{+} \mu^{-} \rightarrow c \bar{c}$ (orange).

$$
\sigma_{h \gamma}^{\text {cut }} \approx 0.53 \mathrm{ab}\left(\frac{\Delta a_{\mu}}{3 \times 10^{-9}}\right)^{2}, \quad \sigma_{Z \gamma}^{\text {cut }} \approx 82 \mathrm{ab}
$$

Requiring at least one jet to be tagged as a $b$, and assuming a $b$-tagging efficiency $\epsilon_{b}=80 \%$, we find that a value $\Delta a_{\mu}=3 \times 10^{-9}$ can be tested at $95 \%$ C.L. at a $30 \mathrm{TeV}$ collider if the probability of reconstructing a $Z$ boson as a Higgs is less than $10 \%$. The resulting number of signal events is $N_{S}=22$, and $N_{S} / N_{B}=0.25$. In Fig. 2 we show as a black line the $95 \%$ C.L. reach from $\mu^{+} \mu^{-} \rightarrow h \gamma$ on the anomalous magnetic moment as a function of the collider energy. Note that since the number of signal events scales as the fourth power of the center-of-mass energy, only a collider with $\sqrt{s} \gtrsim 30 \mathrm{TeV}$ will have the sensitivity to test the $g-2$ anomaly.

The Z-dipole operator $O_{e Z}=\left(\bar{\ell}_{L} \sigma_{\mu \nu} e_{R}\right) H Z^{\mu \nu}$ contributes to $\Delta a_{\mu}$ at one loop, and generates also the process $\mu^{+} \mu^{-} \rightarrow Z h$ (see Fig. 1) with the same cross section of Eq. (5) with $\gamma \leftrightarrow Z$, so that

$$
\sigma_{Z h} \approx 38 \mathrm{ab}\left(\frac{\sqrt{s}}{10 \mathrm{TeV}}\right)^{2}\left(\frac{\Delta a_{\mu}}{3 \times 10^{-9}}\right)^{2} .
$$

Here we assume that only $O_{e Z}$ contributes to $\Delta a_{\mu}$ : it should be stressed that this corresponds to an unnatural scenario, where the coefficients $C_{e B}$ and $C_{e W}$ conspire to cancel out the tree-level contribution from $O_{e \gamma}$. It is nevertheless meaningful to derive the constraint from high-energy scattering on the $Z$-dipole contribution to the $g-2$. The cross section in Eq. (10) has to be compared to the SM irreducible background given by $\sigma_{Z h}^{\mathrm{SM}} \approx 122 \mathrm{ab}\left(\frac{10 \mathrm{TeV}}{\sqrt{s}}\right)^{2}$. Considering again the $h \rightarrow b \bar{b}$ channel, together with hadronic decays of the $Z$, one gets the $95 \%$ C.L. limit shown in Fig. 2 as a blue line.

Next, we derive the constraints on the semi-leptonic operators. The operator $O_{T}^{\mu t}$ that enters $\Delta a_{\mu}$ at one loop can be probed by $\mu^{+} \mu^{-} \rightarrow t \bar{t}$ (see Fig. 1). Its contribution to the cross section is

$\sigma_{t \bar{t}}=\frac{s}{6 \pi} \frac{\left|C_{T}^{\mu t}\right|^{2}}{\Lambda^{4}} N_{c} \approx 58 \mathrm{ab}\left(\frac{\sqrt{s}}{10 \mathrm{TeV}}\right)^{2}\left(\frac{\Delta a_{\mu}}{3 \times 10^{-9}}\right)^{2}$

where in the last equality we have again taken $\Lambda \approx 100 \mathrm{TeV}$ so that $\left|\Delta a_{\mu}\right| \approx 3 \times 10^{-9}(100 \mathrm{TeV} / \Lambda)^{2}\left|C_{T}^{\mu t}\right|$. We estimate the reach on $\Delta a_{\mu}$ simply assuming an overall $50 \%$ efficiency for reconstructing the top quarks, and requiring a statistically significant deviation from the $\mathrm{SM} \mu^{+} \mu^{-} \rightarrow t \bar{t}$ background, which has a cross section $\sigma_{t \bar{t}}^{\mathrm{SM}} \approx 1.7 \mathrm{fb}\left(\frac{10 \mathrm{TeV}}{\sqrt{s}}\right)^{2}$. Similarly, if the charm-loop contribution dominates, we can probe $\left|\Delta a_{\mu}\right| \approx 3 \times 10^{-9}(10 \mathrm{TeV} / \Lambda)^{2}\left|C_{T}^{\mu c}\right|$ through the process $\mu^{+} \mu^{-} \rightarrow c \bar{c}$. In this case, unitarity constraints on the NP coupling $C_{T}^{\mu c}$ require a much lower NP scale $\Lambda \lesssim 10 \mathrm{TeV}$, so that our effective theory analysis will only hold for lower center-of-mass energies. Combining Eqs. (3) and (11), with $c \leftrightarrow t$, we find that

$$
\sigma_{c \bar{c}} \approx 100 \mathrm{fb}\left(\frac{\sqrt{s}}{3 \mathrm{TeV}}\right)^{2}\left(\frac{\Delta a_{\mu}}{3 \times 10^{-9}}\right)^{2} .
$$

The SM cross section for $\mu^{+} \mu^{-} \rightarrow c \bar{c}$ at $\sqrt{s}=3 \mathrm{TeV}$ is $\sim 19 \mathrm{fb}$. In Fig. 2 we show the 95\% C.L. constraints on the top and charm contributions to $\Delta a_{\mu}$ as red and orange lines, respectively, as a function of the collider energy. Notice that the charm contribution can be probed already at $\sqrt{s}=1 \mathrm{TeV}$, while the top contribution can be probed at $\sqrt{s}=10 \mathrm{TeV}$. The simultaneous constraints on the NP couplings $C_{e \gamma}^{\mu}$ and $C_{T}^{\mu t}$ are shown in Fig. 3 for a $30 \mathrm{TeV}$ collider.

So far, we assumed $C P$ conservation. If however the coefficients $C_{e \gamma}, C_{e Z}$ or $C_{T}$ are complex, the muon electric dipole moment (EDM) $d_{\mu}$ is unavoidably generated. Since

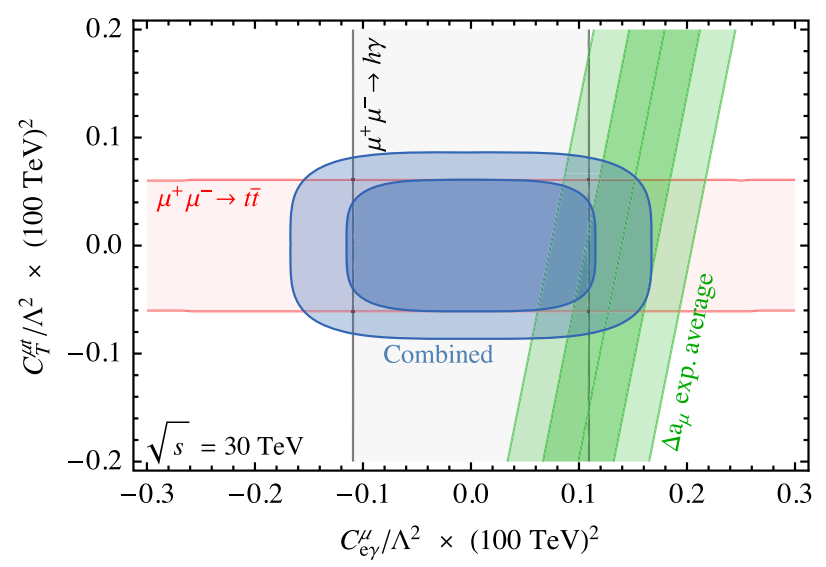

FIG. 3. Constraints on the Wilson coefficients $C_{e \gamma}^{\mu}$ and $C_{T}^{\mu t}$ from $\mu^{+} \mu^{-} \rightarrow h \gamma$ and $\mu^{+} \mu^{-} \rightarrow t \bar{t}$ at a muon collider with $\sqrt{s}=30 \mathrm{TeV}$. The shaded regions are $68 \%$ and $95 \%$ C.L. contours, the individual $1 \sigma$ limits are also shown. 
the cross sections in Eq. (5) and (11) are proportional to the absolute values of the same coefficients, a MC offers a unique opportunity to test also $d_{\mu}$. The current experimental limit $d_{\mu}<1.9 \times 10^{-19} e \mathrm{~cm}$ was set by the BNL E821 experiment [19] and the new E989 experiment at Fermilab aims to decrease this by two orders of magnitude [20]. Similar sensitivities could be reached also by the J-PARC $g-2$ experiment [21].

From the model-independent relation [17]

$$
\frac{d_{\mu}}{\tan \phi_{\mu}}=\frac{\Delta a_{\mu}}{2 m_{\mu}} e \simeq 3 \times 10^{-22}\left(\frac{\Delta a_{\mu}}{3 \times 10^{-9}}\right) e \mathrm{~cm},
$$

where $\phi_{\mu}$ is the argument of the dipole amplitude, the bounds on $\Delta a_{\mu}$ in Fig. 2 can be translated into a modelindependent constraint on $d_{\mu}$. We find that already a $10 \mathrm{TeV}$ $\mathrm{MC}$ can reach a sensitivity comparable to the ones expected at Fermilab [20] and J-PARC [21], while at a $30 \mathrm{TeV}$ collider one gets the bound $d_{\mu} \lesssim 3 \times 10^{-22} e \mathrm{~cm}$.

\section{RARE HIGGS DECAYS}

We finally discuss the connection between the lepton $g-2$ and the radiative Higgs decays $h \rightarrow \ell^{+} \ell^{-} \gamma$. Due to the large luminosity, and the growth with energy of the vector-boson-fusion cross section, a huge number of Higgs bosons is expected to be produced at a high-energy lepton collider [15]. In particular, a MC running at $\sqrt{s}=30 \mathrm{TeV}$ with an integrated luminosity of $90 \mathrm{ab}^{-1}$ will produce $\mathcal{O}\left(10^{8}\right)$ Higgs bosons. With the precision of Higgs couplings measurements most likely limited by systematic errors, the main advantage of having such a large number of events is the possibility to look for very rare decays of the Higgs.

The dipole operator $O_{e \gamma}$ contributes to $h \rightarrow \ell^{+} \ell^{-} \gamma$ as

$\Gamma\left(h \rightarrow \ell^{+} \ell^{-} \gamma\right)_{\mathrm{NP}}=\frac{m_{h}^{3} m_{\ell}}{64 \pi^{3} v} \frac{\operatorname{Re}\left(C_{e \gamma}^{\ell}\right)}{\Lambda^{2}}+\frac{m_{h}^{5}}{768 \pi^{3}} \frac{\left|C_{e \gamma}^{\ell}\right|^{2}}{\Lambda^{4}}$,

where the first term comes from the interference with the SM tree-level amplitude. Combining this expression with eq. (3) gives $\mathcal{B}\left(h \rightarrow \mu^{+} \mu^{-} \gamma\right)_{\mathrm{NP}} \approx 5 \times 10^{-10}\left(\frac{\Delta a_{\mu}}{3 \times 10^{-9}}\right)$, and thus the current muon $g-2$ anomaly cannot be tested at a MC through the process $h \rightarrow \mu^{+} \mu^{-} \gamma$. Instead, $\mathcal{B}\left(h \rightarrow \tau^{+} \tau^{-} \gamma\right)_{\mathrm{NP}} \approx 10^{-5}\left(\frac{\Delta a_{\tau}}{5 \times 10^{-5}}\right)$, and a sensitivity to $\Delta a_{\tau}$ of order $\Delta a_{\tau} \lesssim 5 \times 10^{-5}$ could be attained by measuring $h \rightarrow \tau^{+} \tau^{-} \gamma$ with percent precision [22].

The operator $O_{e Z}$ affects the $h \rightarrow \ell^{+} \ell^{-} Z$ decay in a way analogous to Eq. (14). While the contribution in the $h \rightarrow$ $\mu^{+} \mu^{-} Z$ channel is still too small to be observed, a measurement of $\mathcal{B}\left(h \rightarrow \tau^{+} \tau^{-} Z\right)$ below the percent level could be sensitive to values of $\Delta a_{\tau} \approx 10^{-5}$. It is worth pointing out that at a high-energy lepton collider $\Delta a_{\tau}$ can also be efficiently probed through the processes $\mu^{+} \mu^{-} \rightarrow \tau^{+} \tau^{-}$, and especially $\mu^{+} \mu^{-} \rightarrow \mu^{+} \mu^{-} \tau^{+} \tau^{-}\left(\bar{\nu} \nu \tau^{+} \tau^{-}\right)$which enjoys a very large cross section driven by vector-boson-fusion [22].

\section{CONCLUSIONS}

The muon $g-2$ discrepancy is one of most intriguing hints of new physics emerged so far in particle physics, which has recently been reinforced with the confirmation of the BNL result [1] by the E989 experiment at Fermilab [2]. However, these low-energy determinations of $\Delta a_{\mu}$ rely on the assumption that systematic and hadronic uncertainties are under control at the outstanding level of $\Delta a_{\mu} \sim 10^{-9}$. Therefore, an independent test of $\Delta a_{\mu}$, not contaminated by the above sources of uncertainty, is very desirable.

In this work, we have demonstrated that a muon collider running at center-of-mass energies of several $\mathrm{TeV}$ can achieve this goal, providing a unique, model-independent test of new physics in the muon $g-2$ through the study of the high-energy processes $\mu^{+} \mu^{-} \rightarrow h \gamma, h Z, q \bar{q}$. In particular, a $30 \mathrm{TeV}$ collider with the baseline integrated luminosity of $90 \mathrm{ab}^{-1}$ would be able to reach a sensitivity to the electromagnetic dipole operator of few $\times 10^{-9}$, comparable to the present value of $\Delta a_{\mu}$. If on the other hand the $g-2$ anomaly arises at loop-level from quark-lepton interactions, this could already be tested at a few $\mathrm{TeV}$ collider. Furthermore, we have shown that the current bound on the muon electric dipole moment can be improved by three orders of magnitude, down to few $\times 10^{-22} e \mathrm{~cm}$.

These results rely on measurements with $\mathcal{O}(1)$ accuracy, and thus do not require a precise control of systematic or theoretical uncertainties. We stress that our findings are completely model-independent, being formulated in terms of the very same effective operators that control the lepton dipole moments. Should the muon $g-2$ anomaly be confirmed by forthcoming investigations, this would constitute a no-lose theorem for a multi-TeV muon collider, guaranteeing the discovery of new physics directly in highenergy collisions. Our results add a relevant piece to the already far-reaching potential of a muon collider in highenergy physics.

\section{ACKNOWLEDGMENTS}

We thank M. Passera and A. Wulzer for useful discussions. The work of D. B. was supported in part by MIUR under contract No. PRIN 2017L5W2PT, and by the INFN grant 'FLAVOR'. P. P. acknowledges partial support by FP10 Innovative Training Network Elusives (Grant No. H2020-MSCA-ITN-2015-674896) and InvisiblesPlus (Grant No. H2020-MSCA-RISE-2015-690575). 
[1] G. W. Bennett et al. (Muon g-2 Collaboration), Phys. Rev. D 73, 072003 (2006).

[2] B. Abi et al. (Muon g-2 Collaboration), Phys. Rev. Lett. 126, 141801 (2021); T. Albahri et al. (Muon g-2 Collaboration), Phys. Rev. A 103, 042208 (2021); Phys. Rev. D 103, 072002 (2021).

[3] T. Aoyama et al., Phys. Rep. 887, 1 (2020).

[4] M. Abe et al., Prog. Theor. Exp. Phys. 2019, $053 \mathrm{C} 02$ (2019).

[5] Recently, a lattice QCD collaboration computed the leading hadronic contribution to the muon $g-2$ finding a larger value which weakens the discrepancy with the experimental result to $1.6 \sigma$ [6]. However, this increase to the hadronic contribution could lead to tensions with the electroweak fit or low-energy $e^{+} e^{-} \rightarrow$ hadron data [7]. Therefore, the results of Ref. [6] should be confirmed or refuted by other lattice QCD studies which are underway. Furthermore, there is also the MUonE experimental proposal at CERN aiming to measure the leading hadronic contribution to the muon $g-2$ via $\mu e$ scattering [8].

[6] S. Borsanyi, Z. Fodor, J. N. Guenther, C. Hoelbling, S. D. Katz, L. Lellouch, T. Lippert, K. Miura, L. Parato, K. K. Szabo et al., Nature (London) 593, 51 (2021).

[7] M. Passera, W. J. Marciano, and A. Sirlin, Phys. Rev. D 78, 013009 (2008); A. Keshavarzi, W. J. Marciano, M. Passera, and A. Sirlin, Phys. Rev. D 102, 033002 (2020); A. Crivellin, M. Hoferichter, C. A. Manzari, and M. Montull, Phys. Rev. Lett. 125, 091801 (2020); G. Colangelo, M. Hoferichter, and P. Stoffer, J. High Energy Phys. 02 (2019) 006; Phys. Lett. B 814, 136073 (2021).

[8] G. Abbiendi et al., Eur. Phys. J. C 77, 139 (2017); C. M. Carloni Calame, M. Passera, L. Trentadue, and G. Venanzoni, Phys. Lett. B 746, 325 (2015); P. Banerjee et al., Eur. Phys. J. C 80, 591 (2020).

[9] W. J. Marciano, A. Masiero, P. Paradisi, and M. Passera, Phys. Rev. D 94, 115033 (2016).

[10] W. Buchmuller and D. Wyler, Nucl. Phys. B268, 621 (1986); B. Grzadkowski, M. Iskrzynski, M. Misiak, and J. Rosiek, J. High Energy Phys. 10 (2010) 085; E. E. Jenkins, A. V. Manohar, and M. Trott, J. High Energy Phys.
01 (2014) 035; R. Alonso, E. E. Jenkins, A. V. Manohar, and M. Trott, J. High Energy Phys. 04 (2014) 159.

[11] If the underlying NP generating the dipole moments is also responsible for the generation of the lepton masses, then the chiral enhancement for the dipoles is not at work and the involved scales to explain the muon $g-2$ anomaly have to be of the order of a few $\mathrm{TeV}$.

[12] R. Capdevilla, D. Curtin, Y. Kahn, and G. Krnjaic, Phys. Rev. D 103, 075028 (2021).

[13] J. P. Delahaye, M. Diemoz, K. Long, B. Mansoulié, N. Pastrone, L. Rivkin, D. Schulte, A. Skrinsky, and A. Wulzer, arXiv:1901.06150.

[14] M. Bogomilov et al. (MICE Collaboration), Nature (London) 578, 53 (2020); Bartosik et al., J. Instrum. 15, P05001 (2020); M. Boscolo, J. P. Delahaye, and M. Palmer, Rev. Accel. Sci. Tech. 10, 189 (2019).

[15] A. Costantini, F. De Lillo, F. Maltoni, L. Mantani, O. Mattelaer, R. Ruiz, and X. Zhao, J. High Energy Phys. 09 (2020) 080; D. Buttazzo, D. Redigolo, F. Sala, and A. Tesi, J. High Energy Phys. 11 (2018) 144; S. Dawson and J. L. Rosner, Phys. Lett. 148B, 497 (1984); G. Altarelli, B. Mele, and F. Pitolli, Nucl. Phys. B287, 205 (1987); W. Kilian, M. Kramer, and P. M. Zerwas, Phys. Lett. B 373, 135 (1996); J. F. Gunion, T. Han, and R. Sobey, Phys. Lett. B 429, 79 (1998).

[16] The electron $g-2$ has been invoked in Ref. [17] as a test of the muon $g-2$ anomaly in NP scenarios where the leptonic $g-2$ do not follow a quadratic scaling with lepton masses.

[17] G. F. Giudice, P. Paradisi, and M. Passera, J. High Energy Phys. 11 (2012) 113.

[18] A. Abbasabadi, D. Bowser-Chao, D. A. Dicus, and W. W. Repko, Phys. Rev. D 52, 3919 (1995); A. Djouadi, V. Driesen, W. Hollik, and J. Rosiek, Nucl. Phys. B491, 68 (1997).

[19] G. W. Bennett et al. (Muon (g-2) Collaboration), Phys. Rev. D 80, 052008 (2009).

[20] R. Chislett (Muon g-2 Collaboration), EPJ Web Conf. 118, 01005 (2016).

[21] T. P. Gorringe and D. W. Hertzog, Prog. Part. Nucl. Phys. 84, 73 (2015).

[22] D. Buttazzo and P. Paradisi (to be published). 